\title{
Impact of Combined Preoperative Three-Dimensional Computed Tomography and Intraoperative Real-Time Three-Dimensional Ultrasonography on Liver Surgery
}

\author{
Mitsuo Shimada, M.D. ${ }^{1}$, Takayuki Hamatsu, M.D. ${ }^{1}$, Tatsuya Rikimaru, M.D. ${ }^{1}$, \\ Yo-ichi Yamashita, M.D. ${ }^{1}$, Shinji Tanaka, M.D. ${ }^{1}$, Ken Shirabe, M.D. ${ }^{1}$, \\ Horoshi Honda, M.D. ${ }^{2}$, Makoto Hashizume, M.D. ${ }^{3}$, Keizo Sugimachi, M.D. ${ }^{1}$ \\ ${ }^{1}$ The Department of Surgery II, Faculty of Medicine, Kyushu University, \\ Fukuoka 812-8582, Japan. \\ 2 The Department of Radiology, Faculty of Medicine, Kyushu University,
} Fukuoka 812-8582, Japan

3 The Department of Disaster and Emergency Medicine, Graduate School of Medical Sciences, Kyushu University, Fukuoka 812-8582, Japan

\begin{abstract}
Objective: The aim of this study was to clarify the impact of combined preoperative three dimensional computed tomography (3D-CT) imaging and intraoperative real-time 3D-ultrasonography (US) imaging on image navigation for hepatic resection. Patients and Methods: An integrated navigation system using combined preoperative 3D-CT images and intraoperative real-time 3D-US images were used with patients who underwent hepatic resection for liver tumors, including HCC, cholangiocarcinoma and metastatic liver cancer, and for donor hepatectomy for living related liver transplantation. 3D-CT imaging was made using the workstation "ZIO M900" (ZIO software, Inc., Tokyo, Japan). 3D-US was made in real-time during an operation using the ultrasonographic device SSD5500 (ALOKA, Tokyo, Japan) and the workstation "SAS 200"(ALOKA, Tokyo, Japan). Results: The 3D-CT imaging from CT during hepatic arteriography gave us details of the arteries feeding the tumor. By 3D-CT imaging from CT during arterial portography, the recognition of Glisson's branches in the liver, as well as the hepatic venous system, was much easier
\end{abstract}


than conventional 2D-CT imaging. 3D-US images allowed us to understand the anatomy of the liver more easily than from 2D-US images, and the 3D-US images could reinforce in real-time preoperative information using 3D-CT images. Conclusions: Combined preoperative 3D-CT and intraoperative realtime 3D-US provides us with integrated information of liver anatomy, especially the spatial relationship between tumor and intrahepatic vessels, which can not be visualized because of their location inside of the liver. Intraoperative 3D-US imaging can reinforce preoperative excellent $3 \mathrm{D}-\mathrm{CT}$ imaging. Therefore, our integrated navigation system consisting of combined 3D-CT and 3D-US is useful for liver surgery.

\section{Introduction}

Recent medical advances have made hepatic resections, especially for hepatocellular carcinoma (HCC), much safer than before [1-3]. However, hepatic resections remain one of the most difficult operations in general surgery. Important vessels are hard to be visualized inside the liver, as with brain. Furthermore, in surgery for liver tumors, especially HCC, most livers have chronic liver diseases such as liver cirrhosis. Therefore, the tumor must be resected judging from both anatomical and functional viewpoints. In other words, liver parenchyma including the tumor has to be resected minimally and necessarily, and is often removed in units of less than the segment classified by Couinaud [4], as systemically as possible. Several authors have reported the efficacy of preoperative surgical planning [5-8] in preoperative three-dimensional computed tomography (3D-CT), . In contrast to the great merit of 3D-CT images, the anatomy of the liver is altered in hepatic surgery because of pre-resectional mobilization of the liver from the retroperitoneum. Therefore, during hepatic resections, the efficacy of preoperative planning by 3D-CT images is reduced, and a real-time navigation method has to be developed. Intraoperative ultrasonography (US) has been routinely used during hepatic resections to better understand vessels inside the liver in real-time. Intraoperative US provides a great deal of information about tumor location and vascular anatomy in the liver, and is an indispensable procedure for hepatic resections. However, an accurate three-dimensional understanding of US images depends on the operator's skill and experience, as the original display is twodimensional. Three-dimensional ultrasonography (3D-US) is, therefore, expected to be useful for liver surgery. However, little has been reported on the use of 3D-US during surgery, especially liver surgery [9]. 
The aim of this study was to clarify the impact of combined preoperative 3D-CT imaging and intraoperative real-time 3D-US imaging on image navigation for hepatic resections.

\section{Patients}

Up to now, we have used a combination of preoperative 3D-CT images and intraoperative real-time 3D-US images in cases that have underwent hepatic resection for liver tumors, including HCC, cholangiocarcinoma and metastatic liver cancer, and for donor hepatectomy for living related liver transplantation, at the Department of Surgery II, Kyushu University.

\section{Methods}

\subsection{Definition of Anatomy of the Liver}

The definition of liver segment was that of Couinaud [4], and the anatomic division of the liver of less than Couinaud's segment was defined by the classification of Takayasu et al [10].

\subsection{Preoperative 3D-CT}

Helical CT images of CT arteriography (CTA) and CT during arterial portography (CTAP) were obtained using an X Vigor scanner (Toshiba, Tokyo, Japan) with a section thickness of $3 \mathrm{~mm}$ and a pitch of $3 \mathrm{~mm}(150 \mathrm{~mA}, 130 \mathrm{kV}, 512 \times 512$ matrix) [11]. For CTAP, $100 \mathrm{ml}$ of iopamidol contrast medium (Iopamiron 150; Nihon Schering, Osaka, Japan) was administered through a catheter in the superior mesenteric artery at an estimated rate of $2.5 \mathrm{ml} / \mathrm{sec}$, and sequential helical scanning of the liver was begun 25 seconds after biginning the injection. For CTA, $30-50 \mathrm{ml}$ of Iopamiron 150 was administered through a catheter in the proper hepatic atery at an estimated rate of $1.5 \mathrm{ml} / \mathrm{sec}$, and sequential helical scanning of the liver was begun 5 seconds after biginning the injection. Both 3D CTAP and 3D-CTA imaging were reconstructed from digital data of two-dimensional CT images, and 3D-CTA imaging was constructed from two-dimensional CTA images. 3D-CT imaging was quickly made using the workstation "ZIO M900" (ZIO software, Inc., Tokyo, Japan: http://www.zio.co.jp). 


\subsection{Intraoperative Real-Time 3D-US}

The 3D-US was made in real-time during an operation using the ultrasonographic device SSD5500 (ALOKA, Tokyo, Japan) and the workstation "SAS 200"(ALOKA, Tokyo, Japan). The principle of the SAS 200 is to recognize the 3D-position of the US probe as a $3 \mathrm{D}$ field in the magnetic fie' $A r_{\omega}$ ing a magnetic-field generator. The 3D-US image was generated by the volume-rendering method.

\subsection{Assessment}

Both preoperative 3D-CT and intraoperative real-time 3D-US images were assessed by experienced hepati · curgeons and inexperienced doctors. The points of assessment were both quality of tıu images and accuracy of the spatial recognition of tumor and intrahepatic vessels.

\section{Results}

\subsection{D-CT}

The 3D-CT imaging from CTA gave us a details of the arteries feeding the tumor (Fig. 1). Furthermore, the 3D-CTA image was superior to 2D-hepatic arteriography. Using 3D-CT imaging from CTAP, recognition of Glisson's branches in the liver, as well as hepatic venous system, was much easier than with conventional 2D-CT imaging (Fig.2). By adding colors to each structure, such as portal vein, hepatic artery and hepatic vein, intrahepatic tissue structures were easily visualized. Those 3D-CT images from CTA and CTAP allowed us to plan details of operations.

\subsection{Real-Time 3D-US}

Constructing 3D-US imaging using the workstation "SAS 200" took approximately 3 min. A color doppler mode image was useful to recognize the direction of the blood flow (both inflow and outflow vessels), but the quality of 3D-US images using the color doppler mode was inferior to the images using a power doppler mode, which was excellent to better understand the whole image of the tumor itself and the surrounding structures.Using a power-doppler mode, the vascular system in the liver became more real, and the relationship between tumor and vessels were well visualized in real time. Such 3D-US images allowed us to understand the anatomy in the liver more easily than with 2D-US images (Fig. 3). 


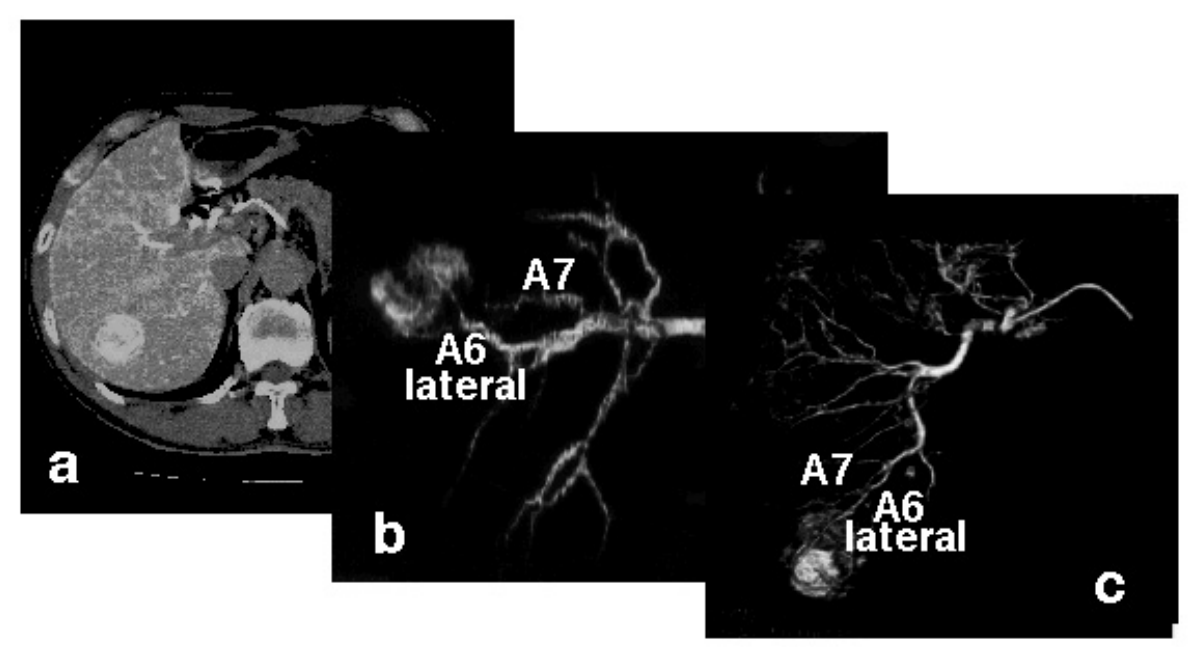

Fig. 1. Preoperative 2D- and 3D-CT images from CT arteriogaraphy (CTA). The 3D-CTA provides an excellent view of the artery feeding the tumor, and is superior to both 2D CTA and 2D-hepatic arteriography. a. 2D-CTA, b. 3D-CTA (right oblique view), c. 3D-CTA (view from foot side)

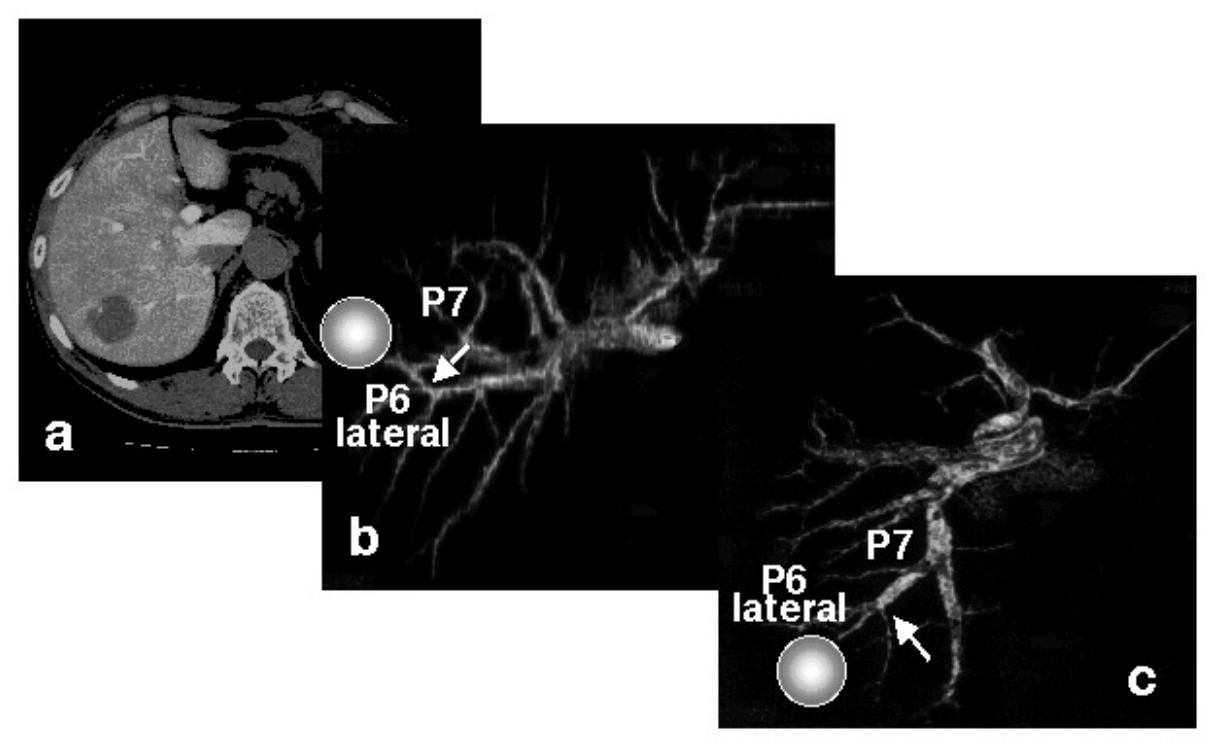

Fig. 2. Preoperative 2D- and 3D-CT images from CT during arterial portography (CTAP). The 3D-CTAP provides excellent information to understand the spatial relationship between tumor and neighboring vessels more easily (Glisson's branches and hepatic veins). An arrow indicates the dividing point, which was planned before an operation.

a. 2D-CTA, b. 3D-CTAP (right oblique view), c. 3D-CTAP (view from foot side) 
With hyperechoic tumor, visualization of both tumor and feeding and/or draining vessels was easy. However, with iso- or hypo-echoic masses, simultaneous views of both tumor and vascular system were difficult. The 3D-US images reinforced in real-time preoperative information using 3D-CT images.

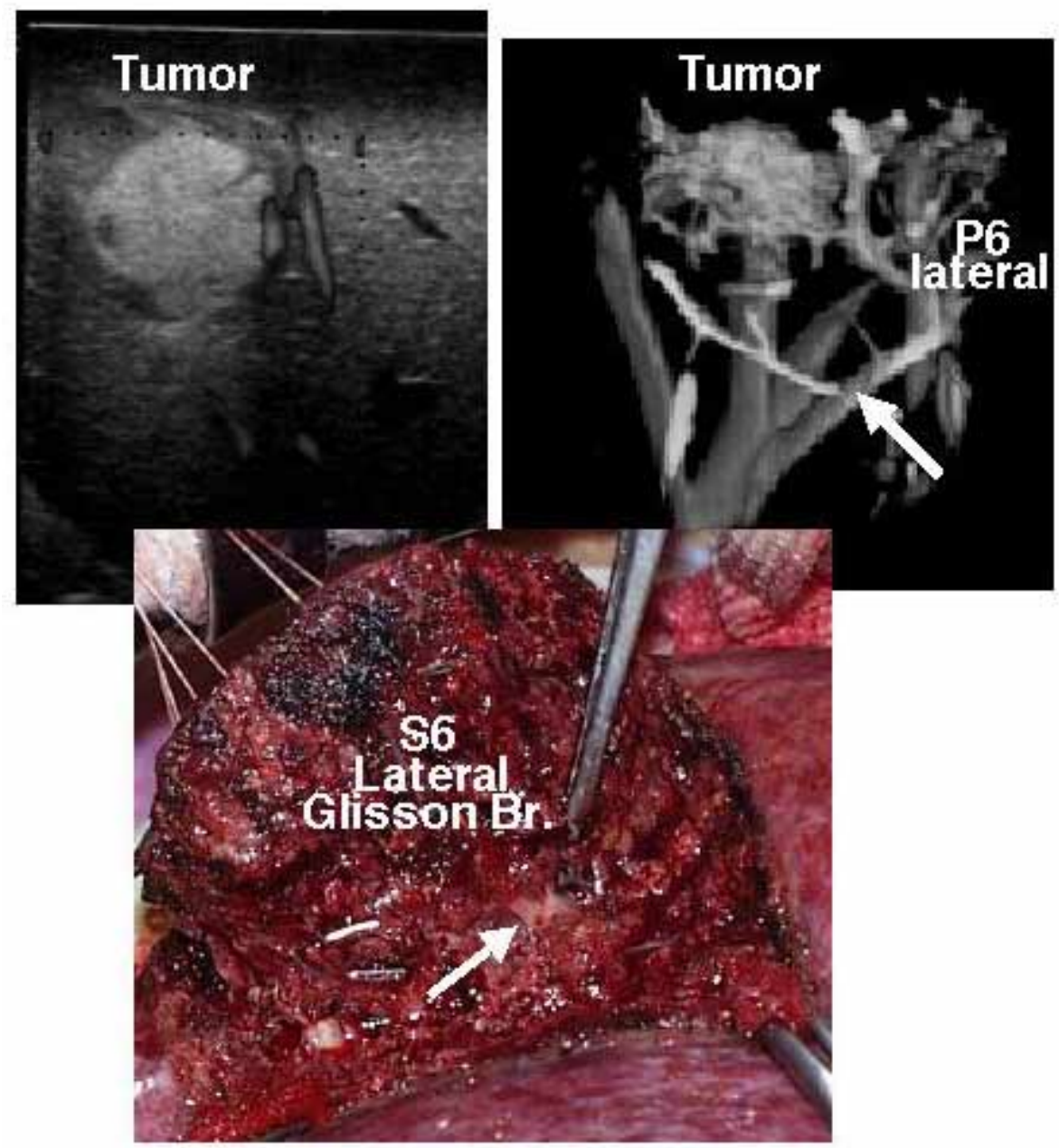

Fig. 3. Intraoperative real-time 2D-US and 3D-US images and intraoperative photography. 2DUS shows a hyperechoic mass and the feeding vessels, which are partly shown by power doppler mode. 3D-US provides excellent imaging by which the spatial relationship between tumor and neighboring vessels, including portal veins and hepatic veins, are clearly recognized.

An arrow indicates the dividing point, which was reconfirmed in real-time during an operation. 


\section{Discussion}

This is the first report on an integrated navigation system for liver surgery, which consisted of combined preoperative 3D-CT images and intraoperative real-time 3DUS images. Our system is superior to each preoperative 3D-CT or intraoperative 3DUS. The quality of images of 3D-CT is superior to 3D-US images now, but, the anatomy of the liver is altered in hepatic surgery, because of pre-resectional mobilization of the liver from the retroperitoneum. Therefore, during hepatic resection, the information of preoperative 3D-CT images is reduced. Our system succeeded in integrating both 3D-CT and 3D-US beneficial characteristics.

With 3D-CT of liver, the 3D-CT technique provides a more accurate diagnosis and a more realistic virtual image of a tumor's location in the liver and so makes possible anatomic resection of the liver [6]. Because diagnostic errors can result in such clinical complications as postoperative bile leakage, this is a useful technique for hepatectomy, especially for sub-subsegmentectomy. Three-dimensional CT angiograms, with their global view of the anatomy and the inherent advantage of volumetric rotation of the vascular system, are useful to surgeons and others with limited experience in interpreting axial anatomy [5]. Determining the hepatic arterial anatomy using 3D-CT angiography has already been shown to be clinically useful in patients being evaluated for liver transplantation. Furthermore, 3D visualization of the organ in relation to the pathology is of great help to appreciate the complex anatomy of the liver [7]. Using virtual reality concepts (navigation, interaction, and immersion), surgical planning, training, and teaching of this complex surgical procedure may be possible. The ability to practice a given hand movement repeatedly will revolutionize surgical training, and combining surgical planning and simulation will improve the efficiency of intervention, leading to optimal care delivery.

Smith et al. [8] reported that their preliminary results suggest that dual-phase spiral CT with CT angiography can provide a comprehensive preoperative liver transplant evaluation, supplying the necessary information for patient selection and surgical planning. As a single, minimally invasive examination, this should have a marked impact on patient care by minimizing procedures and avoiding potential complications. With other uses of preoperative 3D-CT, the advantages of 3D-CT cholangiography are low level invasiveness, easily obtained images compared with those obtained with endoscopic retrograde cholangiography (ERC), good opacification, and provision of a three dimensional understanding of the biliary system, especially of the cystic duct [12]. When combined with US and routine liver 
function tests, 3D-CT cholangiography is considered useful to obtain information before laparoscopic cholecystectomy, as it allows the omission of ERC in many patients who have no common bile duct stone.

With 3D-US in liver surgery, minimum and maximum intensity projections can show the arrangement of blood vessels (hepatic veins and portal veins) and hyperechoic regions, respectively [9]. An intensity projection from several viewpoints can reconstruct 3D imaging by cine-display. Moving a probe manually, images are taken and are processed real-time (in about 10 seconds). 3D-US was used with 24 patients undergoing hepatic resection, and allowed easy visualization of the tumors and vascular anatomy. It is an efficient and safe navigation system in liver surgery.

In conclusion, combined preoperative 3D-CT and intraoperative real-time 3D-US provides us integrated information of liver anatomy, especially the spatial relationship between tumor and intrahepatic vessels, which can not be visualized because of their location inside the liver. Intraoperative 3D-US imaging can reinforce preoperative excellent 3D-CT imaging. Therefore, our integrated navigation system consisted of combined 3D-CT and 3D-US is useful for liver surgery.

\section{Acknowledgements}

This study was supported by the Japan Society for the Promotion of Science, Research for the Future Program, Research on Robotic System in General Surgery (Project number: JSPS-RFTF 99I00902)

\section{References}

1. Shimada M, Takenaka K, Gion T, Fujiwara Y, Kajiyama K, Maeda T, Shirabe K, Nishizaki T, Yanaga K, Sugimachi K. Prognosis of recurrent hepatocellular carcinoma : a 10-year surgical experience in Japan. Gastroenterology 111 (1996) 720-726.

2. Shimada M, Takenaka K, Taguchi K, Fujiwara Y, Gion T, Kajiyama K, Maeda T, Shirabe K, Yanaga K, Sugimachi K. Prognostic factors after repeat hepatectomy for recurrent hepatocellular carcinoma.

Ann Surg 227 (1998) 80-85. 
3. Shimada M, Takenaka K, Fujiwara Y, Gion T, Shirabe K, Yanaga K, Sugimachi K. Risk factors linked to postoperative morbidity in patients with hepatocellular carcinoma. Br J Surg 85 (1998) 195-198.

4. Couinaud C. Lobes et segments hepatiques; notes sur l'architecture anatomique et chirurgicale du foie. Press Med 62 (1954) 709-712.

5. Winter TC 3rd, Nghiem HV, Freeny PC, Hommeyer SC, Mack LA.

Hepatic arterial anatomy: demonstration of normal supply and vascular variants with three-dimensional CT angiography. Radiographics 15 (1995) 771-80.

6. Togo S, Shimada H, Kanemura E, Shizawa R, Endo I, Takahashi T, Tanaka K. Usefulness of three-dimensional computed tomography for anatomic liver resection: sub-subsegmentectomy. Surgery 123 (1998) 73-8.

7. Marescaux J, Clement JM, Tassetti V, Koehl C, Cotin S, Russier Y, Mutter D, Delingette H, Ayache N. Virtual reality applied to hepatic surgery simulation: the next revolution. Ann Surg 228 (1998) 627-34.

8. Smith PA, Klein AS, Heath DG, Chavin K, Fishman EK. Dual-phase spiral $\mathrm{CT}$ angiography with volumetric $3 \mathrm{D}$ rendering for preoperative liver transplant evaluation: preliminary observations.

J Comput Assist Tomogr 22 (1998) 868-74.

9. Shimazu M, Wakabayashi G, Ohgami M, Hiroshi H, Kitajima M.

Clinical application of three dimensional ultrasound imaging as intraoperative navigation for liver surgery. Nippon Geka Gakkai Zasshi 99 (1998) 203-7.

10.Takayasu K, Moriyama N, Muramatsu Y, Shima Y, Goto H, Yamada T. Intrahepatic portal vein branches studied by percutaneous transhepatic portography. Radiology 154 (1985) 31-36.

11.Honda H, Tajima T, Kajiyama K, Kuroiwa T, Yoshimitsu K, Irie H, Abe H, Shimada M, Masuda K. Vascular changes in hepatocellular carcinoma: correlation of radiologic and pathologic findings. AJR 173 (1999) 1213-1217.

12.Kinami S, Yao T, Kurachi M, Ishizaki Y. Clinical evaluation of 3D-CT cholangiography for preoperative examination in laparoscopic cholecystectomy. J Gastroenterol 34 (1999) 111-8. 